\section{Sonographic and Power Doppler Evaluation of an Invasive Mole Located in a Cesarean Scar Pregnancy}

We report on the sonographic evaluation, clinical management, and pathologic characteristics of an unusual case of a partial molar cesarean scar pregnancy that evolved into an invasive mole. Initially, this pregnancy was misdiagnosed as a simple cesarean scar pregnancy. After treatment with local and systemic methotrexate (MTX), 2-dimensional sonography and a power Doppler evaluation together with serum $\beta$-human chorionic gonadotropin $(\beta$-hCG) test results were suggestive of a molar pregnancy; a complementary 3-dimensional (3D) sonographic evaluation was also performed to better identify and localize the blood supply to the cesarean scar pregnancy and to measure the volume of the ectopic mass. To our knowledge, the sonographic features of this entity have not been reported previously.

A 34-year-old woman (gravida 2, para 1, 1 previous surgical termination of pregnancy, and 1 urgent cesarean delivery) was referred to our department at a gestational age of 6 weeks 4 days with abdominal pain and vaginal bleeding. The first transvaginal sonographic diagnosis was a cesarean scar pregnancy, showing an empty uterus with a $35 \times 30$-mm complex mass with cystic spaces implanted on the anterior portion of the lower uterine segment and a very thin $(1.5-\mathrm{mm})$ layer of myometrium from the bladder (Figure 1A); 2 days later, an embryo with evidence of cardiac activity (crown-rump length, $3 \mathrm{~mm}$ ) was visualized in the ectopic mass. Doppler velocimetry showed a high peak systolic velocity $(40 \mathrm{~cm} / \mathrm{s})$ and low-impedance (resistive index, 0.3) turbulent blood flow around and inside the ectopic mass. The gestational sac volume and vascularization were assessed by a $3 \mathrm{D}$ ultrasound machine with a 6-12-MHz transvaginal transducer (Voluson E8; GE Healthcare, Milwaukee, WI). Power Doppler settings were a $0.9-\mathrm{kHz}$ pulse repetition frequency and a $200-\mathrm{MHz}$ filter. The chorionic sac volume and vascularization were analyzed by virtual organ computer-aided analysis software (4D View; GE Healthcare). Six rotational steps $\left(60^{\circ}\right.$ apart) were used to define the sac volume, including the vascular "ring" in the perimeter, and the vascularization index (VI; the number of color flow-containing voxels divided by the total number of voxels contained within the volume, expressed as a percentage) was also calculated by the same software.

On admission, the serum $\beta$-hCG concentration was $51,547 \mathrm{IU} / \mathrm{L}$; the $3 \mathrm{D}$ volume of the gestational sac was $8.07 \mathrm{~cm}^{3}$; and the 3D power Doppler VI was $28 \%$.
The same parameters were examined every 10 days from diagnosis to resolution (Figure 2).

After the diagnosis of a cesarean scar pregnancy and counseling about risk, the patient opted for termination of the pregnancy, according to the following procedure: a transvaginal ultrasound-guided puncture, using a 20-gauge needle, in the area of the embryonic heart with potassium chloride injection ( $2 \mathrm{~mL}$ of $2 \mathrm{mEq}$ potassium chloride), followed by aspiration of liquid inside the sac and slow injection of $50 \mathrm{mg}$ of MTX. The patient subsequently received additional 50-mg intramuscular injections of MTX after 10 and 30 days, respectively.

Three weeks after the second systemic MTX injection, the patient returned to our department for severe abdominal pain that radiated to the back. The serum $\beta$ hCG concentration was reduced to $2503 \mathrm{IU} / \mathrm{L}$, whereas the $3 \mathrm{D}$ sonographic volume increased to $141 \mathrm{~cm}^{3}$, and the VI increased to $72 \%$; power Doppler imaging highlighted turbulent blood flow around and inside the ectopic mass (Figure 1B). The lack of reduction of the ectopic mass and vascularization despite the drop in $\beta$-hCG in response to local and systemic therapy and the persistent symptoms of the patient led us to hypothesize a cesarean scar trophoblastic tumor or an arteriovenous neoformation. After counseling, the patient underwent laparotomy, with cautious adhesiolysis and resection of the uterine site of the ectopic mass, which infiltrated the posterolateral isthmic wall and the serosa-bladder wall interface (Figure 1C); surgery terminated with a plastic uterine reconstruction, suturing the myometrial flaps with a continuous double layer. The $\beta$-hCG level dropped to $676 \mathrm{IU} / \mathrm{L}$ on the day after the surgery ( $<10 \mathrm{IU} / \mathrm{L}$ after 2 weeks), and sonography 5 days after the surgery showed no evidence of the previous lesion. The histologic examination showed an invasive mole located on the cesarean scar pregnancy. The patient's follow-up, including a clinical evaluation, pelvic and abdominal sonographic scans, chest radiography, and computed tomography, excluded possible metastatic disease.

Gestational trophoblastic disease in a cesarean scar pregnancy is exceedingly rare, is difficult to manage, and can have potentially catastrophic consequences. No therapeutic protocols have been established about this rare condition, and it is possible that more cases of cesarean scar gestational trophoblastic disease will be encountered in the future, owing to the increasing incidence of cesarean scar pregnancies. So far only 4 cases of cesarean scar gestational trophoblastic disease have been reported in the literature: 1 case of a partial molar pregnancy in a cesarean scar, which was initially misdiagnosed as a threatened abortion ${ }^{1} ; 1$ 

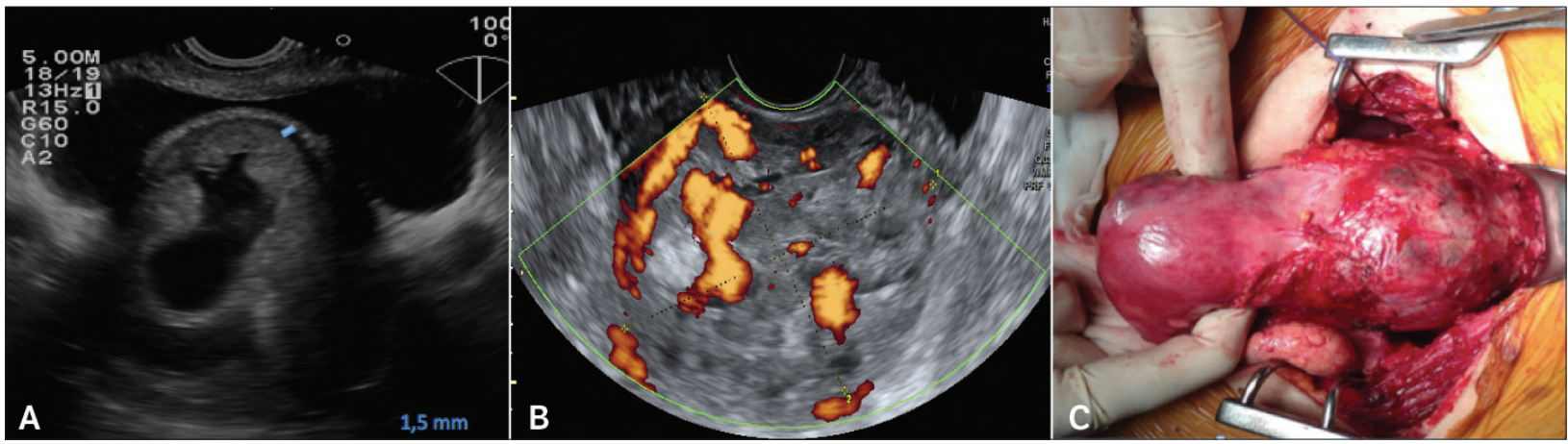

Figure 1. A, Transvaginal sonogram showing a retroverted uterus with a cesarean scar pregnancy surrounded by thin myometrium of $1.5 \mathrm{~mm}$ (blue line). B, Power Doppler sonogram showing turbulent blood flow around and inside the ectopic mass 7 weeks after local treatment. C, Macroscopic features of the invasive mole at surgery.

cesarean scar molar pregnancy with a delayed diagnosis until 10 months later, when the patient presented with vaginal hemorrhage requiring an emergency hysterectomy ${ }^{2} ; 1$ cesarean scar molar pregnancy diagnosed 3 weeks after pregnancy termination at 7 weeks and treated by dilation and curettage and uterine artery embolization to control the ensuing bleeding ${ }^{3}$; and 1 case of choriocarcinoma in a cesarean scar pregnancy treated with preventive uterine artery embolization coupled with an arterial MTX injection before dilation and curettage. ${ }^{4}$ In this last case, at 7 weeks, the $\beta$-hCG level $(312,468 \mathrm{IU} / \mathrm{L})$ and blood flow were much higher than in a normal cesarean scar pregnancy (resistive index, 0.38).
To the best of our knowledge, our case is the first report of a cesarean scar invasive molar pregnancy evaluated by 2 and 3-dimensional sonography and characterized by an atypical $\beta$-hCG resolution curve and 3D volume and vascularization changes in the ectopic mass after local (potassium chloride and MTX) and systemic (MTX) treatment. Indeed, despite a regular fall in the $\beta$-hCG level, there were increases in the volume and vascularization of the ectopic mass and maternal symptoms. Usually after treatment of a cesarean scar pregnancy, initial increases in the serum $\beta$ hCG concentration and gestational sac volume have been reported, but they were quickly followed by a slow decrease ( $\beta$-hCG nondetectable 41-100 days after MTX injection and involution of the gestational sac until 5-6 months). ${ }^{5}$

Figure 2. Graphic representation of 3D volume, VI, and $\beta$-hCG from diagnosis to surgery of the molar cesarean scar pregnancy.

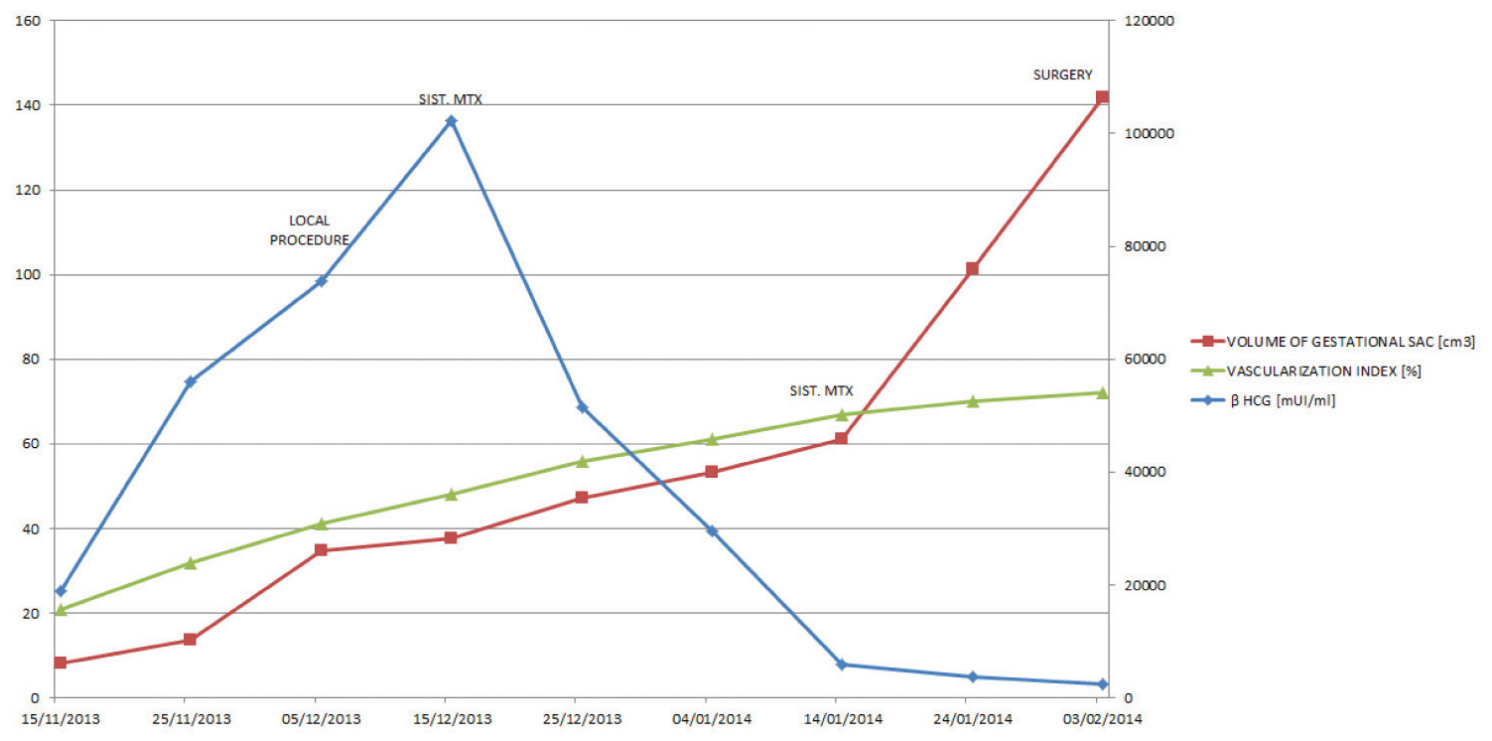


Although a molar pregnancy in a cesarean scar is very difficult to diagnose, the initial abnormally elevated serum $\beta$-hCG levels, persistence of symptoms after primary treatment, increases in the volume of the ectopic mass and vascularization, and cystic changes despite the drop in $\beta$-hCG levels may be useful criteria for diagnosis of gestational trophoblastic disease in a cesarean scar pregnancy.

Antonella Vimercati, MD, PhD, Alessandra Caterina de Gennaro, $M D, P h D$, Leonardo Resta, $M D, P h D$, Gennaro Cormio, $M D$, PhD, Ettore Cicinelli, $M D, P h D$ Department of Obstetrics and Gynecology (A.V., A.C.d.G., G.C., E.C.) Department of Pathology (L.R.) University Hospital Policlinico of Bari University of Bari "Aldo Moro" Bari, Italy

doi:10.7863/ultra.15.08010

\section{References}

1. Wu CF, Hsu CY, Chen CP. Ectopic molar pregnancy in a cesarean scar. Taiwan J Obstet Gynecol 2006; 45:343-345.

2. Michener C, Dickinson JE. Caesarean scar ectopic pregnancy: a single centre case series. Aust NZ J Obstet Gynaecol 2009; 49:451-455.

3. Ko JK, Wan HL, Ngu SF, Cheung VY, Ng EH. Cesarean scar molar pregnancy. Obstet Gynecol 2012; 119:449-451.

4. Qian ZD, Zhu XM. Caesarean scar choriocarcinoma: a case report and review of the literature. EurJMed Res 2014; 19:25.

5. Timor-Tritsch IE, Monteagudo A, Santos R, Tsymbal T, Pineda G, Arslan AA. The diagnosis, treatment, and follow-up of cesarean scar pregnancy. AmJ Obstet Gynecol 2012; 207:44.e1-44.e13. 